\title{
Competence in Teaching Mindfulness-Based Courses: Concepts, Development and Assessment
}

\author{
Rebecca S. Crane • Willem Kuyken • \\ J. Mark G. Williams • Richard P. Hastings • \\ Lucinda Cooper • Melanie J. V. Fennell
}

Published online: 22 September 2011

(C) Springer Science+Business Media, LLC 2011

\begin{abstract}
There has been a groundswell of interest in the UK in Mindfulness-Based Stress Reduction (MBSR) and its derivatives, particularly Mindfulness-Based Cognitive Therapy (MBCT). Many health, education and social work practitioners have sought ways to develop their competencies as mindfulness-based teachers, and increasing numbers of organisations are developing mindfulness-based training programmes. However, the rapid expansion of interest in mindfulness-based approaches has meant that those people offering training for MBSR and MBCT teachers have had to consider some quite fundamental questions about training processes, standards and competence. They also need to consider how to develop a robust professional context for the next generation of mindfulness-based teachers. The ways in which competencies are addressed in the secular mainstream contexts in which MBSR and MBCT are taught are examined to enable a consideration of
\end{abstract}

R. S. Crane $(\bowtie) \cdot$ L. Cooper

Centre for Mindfulness Research and Practice,

School of Psychology, Bangor University,

Bangor, Gwynedd, UK LL57 1UT

e-mail: r.crane@bangor.ac.uk

W. Kuyken

Mood Disorders Centre, School of Psychology,

University of Exeter,

Exeter, UK EX4 4QG

J. M. G. Williams • M. J. V. Fennell

University of Oxford Department of Psychiatry,

Warneford Hospital,

Oxford, UK OX3 7JX

R. P. Hastings

School of Psychology, Bangor University,

Bangor, Gwynedd, UK LL57 2AS the particularities of mindfulness-based teaching competence. A framework suggesting how competencies develop in trainees is presented. The current status of methodologies for assessing competencies used in mindfulness-based training and research programmes is reviewed. We argue that the time is ripe to continue to develop these dialogues across the international community of mindfulness-based trainers and teachers.

Keywords Mindfulness-based approaches · Mindfulnessbased cognitive therapy $\cdot$ Mindfulness-based stress reduction $\cdot$ Professional practice $\cdot$ Training $\cdot$ Assessment . Competence

\section{Introduction}

The rapid expansion of interest in the implementation of mindfulness-based approaches in a diversity of contemporary settings holds both promise and risk. The promise is of accessibility in mainstream secular settings to a contemplative approach which has a long lineage in supporting people to better know the territory of their interior experience, to train their minds in a certain direction and so to radically transform their fundamental understanding of, and approach to life. The risk is that, in the drive to implement a promising approach, the very factors which give rise to its promise are lost through a dilution of its integrity. The quality of a mindfulness-based class is only as good as the instructor and his or her understanding of what is required to deliver a programme which is both rooted in a depth of personal mindfulness practice (Kabat-Zinn 2011) and integrated with the skills and understandings relevant to the particular program or context within which the teaching is offered (Teasdale et al. 2003). 
In the development of Mindfulness-Based Stress Reduction (MBSR) and its many derivatives during the 1980s and $1990 \mathrm{~s}$, and even into the first few years of the new millennium, most teacher trainers were either first generation (that is, they learned through direct contact with the program originator, Jon Kabat-Zinn, and his close colleagues), or second generation (they learned from trainers who themselves had direct contact with the originators). Indeed, most of the positive research results reflect the outcomes of classes taught by these first- and second-generation teachers. We are now in a different phase of development. The enthusiasm to implement secular mindfulness-based programmes in a range of contexts (including healthcare, education, business, prisons and community centres), and with a diversity of client populations within these contexts, is strong and growing. Within the UK, a strong driver to the growth in interest has been the recommendation by the National Institute for Health and Clinical Excellence (NICE; 2004, 2009) of MindfulnessBased Cognitive Therapy (MBCT) as a National Health Service treatment of choice for people with recurrent depression.

In this rapidly developing context, there is a growing demand for teachers and trainers of teachers. Understandably, concerns are being expressed from within the field that practitioners may be tempted to respond to this demand without having engaged in the personal preparation and developed the competencies required for these endeavours (e.g. Grossman 2010; Kabat-Zinn 2011). The abstract for a workshop at the 2011 Annual Conference of the Center for Mindfulness, USA eloquently expresses this concern: 'the drive to train more Mindfulness-Based Stress Reduction (MBSR) professionals runs the risk of easily and inadvertently undermining the integrity, exactness and long-term commitment required for the formation and ongoing development of competent well trained MBSR teachers' (Santorelli et al. 2011).

In the UK, there has been a concerted focus on responding both to the growing demand and to the concerns about quality and integrity since 2006. Three interconnected good practice foundations to quality and integrity in teaching mindfulness-based courses which we are exploring in parallel are: (1) training processes, (2) standards for teachers and (3) mindfulness-based teaching competence (see Fig. 1).

In an initial publication, we articulated the principles underpinning our training processes (Crane et al. 2010). The UK Network for Mindfulness-Based Teacher Trainers, which represents all the main UK training organisations, has developed and disseminated national Good Practice Guidance on standards for mindfulness-based teachers (UK Network of Mindfulness-Based Teacher Trainers 2010). These guidelines focus on the practical implications for teachers of the underlying ethical principles and values of

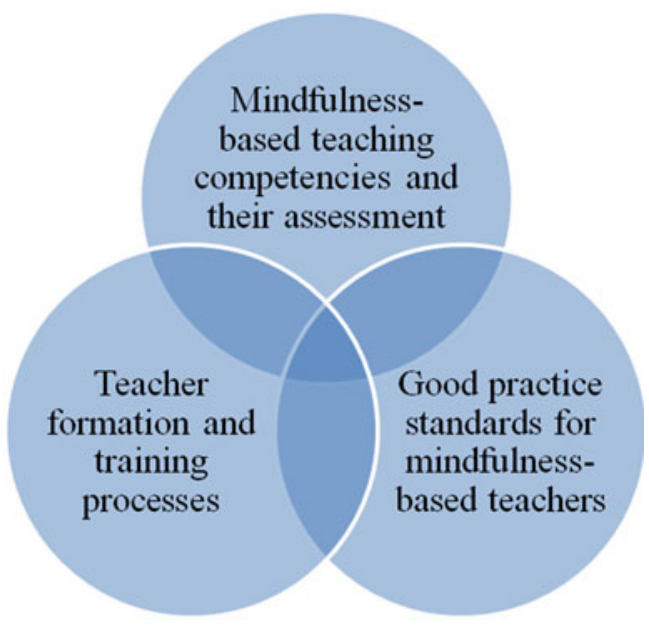

Fig. 1 Working model of three interconnected aspects of quality and integrity in teaching mindfulness-based courses

mindfulness-based teaching. In this article, we focus on the third of these foundations: mindfulness-based teaching competencies. First, we examine the wider context of how competencies are addressed in the secular mainstream contexts in which MBSR and MBCT are taught. Second, we investigate how the conceptualisation of competence and its assessment relate to the field of mindfulness-based teaching. Third, we examine the development of mindfulness-based teaching competencies. Fourth, we review the current status of methodologies for assessing competencies used in mindfulness training and research programmes. The article is set within the context of MBSR and its derivatives, particularly MBCT, which, because of the NICE Guidelines, has a strong influence in the UK.

\section{Competence in Related Fields}

\section{Definitions}

Competence is one component of treatment integrity (the extent to which the approach is carried out as intended); the others being adherence (the extent to which the teacher applies the appropriate 'ingredients' at the appropriate time point and does not introduce intervention procedures which are not recognised as a part of the approach) and treatment differentiation (how the approach can be distinguished from other approaches; Weck et al. 2011). These three components are interrelated, and although our main focus here is competence, we will also refer to adherence and treatment differentiation. 'Competency' and 'competencies' are demonstrated acquisition of specific knowledge, skills and attitudes which added together make up competence to practice. 'Competence' is a broader concept relating to fitness to practice. Competence frameworks are structures 
that set out and define individual areas of competence required by individuals for workplace roles. The articulation of competencies is a statement from an organisation or professional group to individuals wishing to practice in a domain, and also to the wider public about what competencies to expect from individuals. Competence frameworks provide a map of the processes, behaviours and actions that will be valued, recognised and, as appropriate, rewarded (Chartered Institute of Personnel and Development 2011).

Epstein and Hundert (2002) proffered a definition of professional competence for the medical profession which is apt for all those working in service of others: 'competence is the habitual and judicious use of communication, knowledge, technical skills, clinical reasoning, emotions, values, and reflection in daily practice for the benefit of the individual and community being served'. Significantly, this definition underlines that competence relies on habits of mind including attentiveness, curiosity, self-awareness and presence. Competence relates to the knowledge, skills and attitudes relevant to the particular profession and crucially any actions arising from them must be executed in accord with the ethical principles, standards, guidelines and values of the profession (Rodolfa et al. 2005). Competence is developmental since the capability of the individual differs depending on the individual's stage of professional functioning.

\section{Competence in the Workplace}

In psychological therapies, medicine and education, there is a developing emphasis on competency-based education, training and credentialing. Professional groups increasingly delineate their contribution to the field by identifying their foundation, core and specialized competencies. Public verification of competence through professional training programmes provides a system through which a profession can institute boundaries recognised by the general public between those who have engaged in a process of developing particular competencies and have subjected these to the scrutiny of others, and those who have not. The accreditation of professional education and training programmes is based largely on demonstration of how particular competencies are evidenced (Epstein and Hundert 2002; Kaslow 2004).

Some of the current drive to develop competence frameworks comes from government-led initiatives. For example, in the UK "Skills for Health" is undertaking the development of National Occupational Standards for a range of occupations including psychological therapies. A key driver for this is the 'Improving Access to Psychological Therapies' initiative which requires the provision of NICE-approved evidencedbased psychological therapies in England (IAPT 2011). In turn, this has increased the demand and funding for therapist training and required thinking at a national level on the particular competencies required for different levels of psychological intervention and for different psychological modalities (Skills for Health 2011). This included a careful mapping of the domains of competencies required by different types of therapists (e.g., cognitive-behavioural) and suggested methods for assessing these competencies, but MBCT was not included in this process.

\section{Foundational and Specific Competence}

Barber et al. (2007) defined two areas of competence: the broader competencies represented by a particular profession (foundational competence) and those required to carry out specific roles or specialities within the broader context of professional practice. Rodolfa et al. (2005) asserted that the acquisition of speciality competencies should occur later in the training sequence and require more sophisticated credentialing. Here, we focus on the issues related to the development and assessment of the speciality competencies required to teach mindfulness-based approaches. These competencies build on the broader, foundational professional competencies of our trainees and should not be considered in isolation from these foundation competencies.

\section{Competence in the Context of Mindfulness-Based Teaching}

In this section, we examine the questions: How can we best ensure that the conceptualisation and assessment of competence are compatible with the underlying philosophies and values of mindfulness, and serve the integrity of developments in the field? We also discuss how the issue of foundational and speciality competencies relates to mindfulness-based teaching.

\section{Competence and the Underlying Philosophy of Mindfulness-Based Approaches}

The development of a competence framework for mindfulness-based teachers raises the understandable concern that the endeavour represents an attempt to operationalize a process whose life blood is a spontaneous responsiveness to the momentary arising and an experiential understanding of the impermanence of experiences. The underlying philosophy of mindfulness practice is based on the 2,500-year-old tradition of Buddhism which articulates a methodology for engaging in a process of inner exploration or 'interior empiricism' (Owen 1996). Through direct connection with the interplay of internal experience, an exploration of our own nature and the nature of the world around us becomes possible. Underpinning this is the understanding that there are limits to the reach of the 
conceptual mind and that a rethinking of consciousness, awareness and the way the mind processes information is needed - aspects of reality and truth are ungraspable by the narrow limits of most contemporary accounts of the mind (Teasdale and Chaskalson 2011; Williams 2008).

There are clearly some inherent tensions in applying a paradigm of competence to the field of mindfulness-based teaching. For example, the traditional language of workplace competence includes the expression 'performance' of 'roles'. It is not possible to conceive of mindfulness as an 'add-on', or for the teaching process to be seen as a set of techniques, a collection of skills which can be learned. Rather, the "way of being' which emerges through sustained engagement with the practice becomes an integral part of the 'person of the teacher' (McCown et al. 2010), not a role which is performed. A second example of this tension relates to the risk of duality which the language of and focus on competence can create. There can be a shift in perspective towards 'right' and 'wrong', judgement, opinion and a focus on 'my' teaching process and 'my' competence. These mind states lead away from the capacity to be open and connected and towards identification and separation. Whereas, inherent within mindfulness teaching is the message that there are universal aspects to the experience of being human: centrally, that we all experience suffering, which ultimately comes from ignorance about ourselves and the nature of reality. Mindfulness practice leads us to see more clearly the ways we fuel our suffering and opens us to experiencing our connection with others.

The competencies that are under examination in the context of mindfulness teaching are thus that the teacher is able to become a vehicle for conveying this by allowing themselves to be human (and so to be vulnerable), in contrast to holding onto a position of 'expertise' or indeed competence. Clearly, if the need to be competent or to develop competence is in the forefront of a teacher's mind while they are teaching, the capacity to be connected to the participants and the wider perspectives and spaciousness which inform the teaching are likely to be compromised.

Acknowledging these tensions, we also argue that on all levels of experience - intuitively, emotionally, cognitively and viscerally - participants recognise when they are on the receiving end of an authentic, skilful, attuned teaching process. Rather than purporting to define the teaching process, a competence framework for mindfulness teaching can be descriptive of what we see, hear and sense when we are participating in skilful teaching. The various lineages and traditions from which mindfulness arose have long used the conceptual articulation of mindfulness practice to point towards the essence of the process. There is also a literature describing the pedagogy and processes of MBSR and MBCT teaching (Kabat-Zinn et al. 2011; McCown et al. 2010; Santorelli 1999; Segal et al. 2002) which offers a robust basis for developing descriptors of competence.
Competence and the Integrity of Mindfulness-Based Teaching Developments

Issues related to competence impact on all the areas of growth for mindfulness teaching - within training programmes where larger numbers of trainers are supporting and assessing larger numbers of developing teachers; within mainstream contexts such as the UK health service where MBCT is increasingly being commissioned and implemented and governance relating to teacher readiness is required; and within research contexts where methods are needed to assess that the teachers delivering classes in research trials have achieved an appropriate level of competence, that they deliver the intervention with fidelity during the research.

In the initial development of the integration of mindfulness into contemporary settings, issues relating to teacher standards, formation, readiness and assessment were held by a small number of teacher training organisations which were predominantly led by first- and second-generation leaders in the field. The expansion of training programmes creates concerns about potential dilution in integrity. There are inevitable questions about their accountability and the meaning of the credentials that they give to graduates of training.

Expansion creates a new context for the mindfulness-based teaching and training community to be operating within and needs a new response from within the profession. There are risks that if this response is not robust enough, the pressures from outside could inadvertently propel standards in a downward direction. Competencies are understood by mainstream settings, they support communication to service managers and others who are not inside the profession about 'readiness-to-teach', and they help to identify training requirements for developing teachers. The mindfulness field has a responsibility to the public, and to developing trainees, to be continually addressing these issues so that a professional context develops in which there are appropriate boundaries, expectations and guidance.

There is already evidence of an increasing urge from within to develop governance processes which set benchmarks for expected levels of competence, which ensure that mindfulnessbased trainers and teachers who are working with integrity are not undermined by those who are not and which maintain public confidence in the profession. Examples of this trend are the work of the UK Network of Teacher Trainers and the recent publication of standards for trainers of MBSR teachers (Kabat-Zinn et al. 2011). We argue that the time is ripe to continue to develop these dialogues across the international community of mindfulness-based trainers and teachers.

\section{Foundational and Speciality Competencies}

In terms of foundational experience, the mindfulness-based training programmes represented by the authors require 
trainees to normally have a minimum of 3 years independent professional practice prior to embarking on training in mindfulness-based teaching. This is designed to ensure that trainees already have in place the generic professional competencies and experience within their particular context prior to developing specialist mindfulness-based teaching competencies. The differences between MBSR and MBCT are small but important. At the present time, MBCT is most commonly offered in clinical settings or in contexts where an underpinning psychological model is required, or provides a vital framework for understanding the factors that underlie and maintain complex psychological problems. MBCT trainees who plan to use the approach in such clinical settings need a clinical training plus training in the use of evidence-based psychological models and treatment. MBSR is offered in a broader range of settings including clinical, business and educational contexts. MBSR trainees require a professional background and training relevant to the context within which they intend to teach.

Furthermore, as described in our earlier paper on training, prospective teachers are required to have a developed mindfulness practice prior to engaging in teacher training (Crane et al. 2010). In addition to foundational experience, teaching mindfulness-based courses require a range of competencies including working to and within the curriculum, relational skills, guiding mindfulness practices, conveying the teaching themes through both the process and the content of the teaching and holding the 'container' or group context of the teaching. Woven across all these processes is the capacity of the teacher to teach everything through an embodiment of the qualities of mindfulness. 'Embodiment of process' by the teacher is the feature of the approach which differentiates it from other approaches and is therefore illustrative of the particularities which need consideration in developing a mindfulness-based teaching competence framework.

In essence, we are aiming to describe how the interior work of mindfulness practice is tangibly sensed in the MBSR/MBCT classroom. That is, the extent to which the teachers are in mindful connection with their interior direct experience and the interface of this with the group, individual participants and the teaching process. Through the process of inner contemplative exploration, the teacher comes naturally to embody the qualities that are inherent within the exploration itself (e.g. intentional focus of attention, engaged curiosity, equanimity, compassion). Embodiment communicates the essence of the potential which mindfulness offers on a level beyond the conceptual. Teachers are not sharing a sensible philosophical approach to life with their participants; they are participating with them in an exploration which is integral to their own life. In our personal experience, our capacity to embody the essence of mindfulness teachings depends on a continuing 'alive' connection with our own practice and with teachings from the wisdom traditions which gave rise to mindfulness. Of course, a related competence is the capacity to locate the teaching in mainstream secular contexts whilst sustaining connection to and remaining true to the wisdom and understanding inherent within the traditions which gave rise to mindfulness teaching.

\section{How Might Mindfulness-Based Teaching Competence Develop?}

Dreyfus and Dreyfus's (1986) landmark research on the development of competence investigated the process of skills acquisition in human endeavours that require sustained application. They provided evidence that we gain skills through experience, instruction and imitation, and through experiential learning gain the capacity to respond intuitively in complex situations. Through their research on skill acquisition in a range of areas (airplane pilots, chess players, car drivers and adult learners of second languages), they demonstrated that the development of competence is a lifelong endeavour which continues to pass through subtle shifts, and that a person usually moves through characteristic stages as they develop competence. We currently have no evidence that mindfulness-based teaching competence develops in these ways, but we recognise the generic processes identified by Dreyfus and Dreyfus through systematic observation of the developmental process of skills acquisition in ourselves and our trainees. Furthermore, we were influenced by Sharpless and Barber's (2009) reinterpretation of the Dreyfus and Dreyfus work to intervention competence in clinical psychology, which begins to give a language for these processes closer to the context of mindfulness-based teaching. Table 1 offers a summary form of our reinterpretation of these developmental stages in the context of mindfulness-based teaching. To the original five stages, we have added 'incompetent' at the bottom end (to represent the spectrum required in postgraduate assessment), have changed the term 'novice' to 'beginner', and at the top end have changed the term 'expert' to 'advanced' (to minimise some of the problematic connotations of the word expert).

Relationship with Training It is natural for teachers to develop competence over time as skills and understanding develop, and with the appropriate training and good practice processes. 'Beginner' and 'advanced beginner' are appropriate levels for teachers in the early stages of training; 'competent' is an appropriate level for a student teacher graduating from a full teacher training program/ supervised pathway with some early teaching experience in the field; 'proficient' is an appropriate level for a teacher 
Table 1 Adaptation of Drefus and Dreyfus stages of competence (Dreyfus and Dreyfus 1986) to mindfulness-based teaching competence

\begin{tabular}{|c|c|}
\hline Stage & Competence descriptor \\
\hline 1. Incompetent & $\begin{array}{l}\text { Teaching is inappropriate, likely to compromise the safety of participants and to lead to negative therapeutic consequences. } \\
\text { The trainee has not grasped the fundamentals of mindfulness-based teaching practice and does not recognise basic } \\
\text { principles and rules. There is nonadherence to the program form. }\end{array}$ \\
\hline 2. Beginner & $\begin{array}{l}\text { Trainees operate in a state of rule learning and rule governedness. Preparation for teaching is likely to be dominated by } \\
\text { memorising scripted responses to participants and bringing to mind a list of 'teaching points' to bring to class dialogues. } \\
\text { Although aspects of competence are demonstrated at this stage, there are numerous substantive problems and an overall } \\
\text { lack of consistency. Experience is needed to progress through this stage-underlining the importance of training } \\
\text { programmes offering considerable time to practice skills with fellow trainees and receive feedback. }\end{array}$ \\
\hline $\begin{array}{l}\text { 3. Advanced } \\
\text { beginner }\end{array}$ & $\begin{array}{l}\text { Having had opportunities to practice newly acquired skills in mindfulness-based teaching, trainees are able to more easily } \\
\text { recall the 'rules' that need applying (i.e. the different steps within the sitting practice guidance, ways of opening up class } \\
\text { dialogue following a practice) and are increasingly able to apply these in a more sophisticated way. The teaching process } \\
\text { remains deliberative, and there are significant inconsistencies that require further development, but competencies are } \\
\text { clearly demonstrated. Participants' emotional and physical safety is adequately taken care of, and at a very basic level, the } \\
\text { teacher is 'fit for practice' - at this stage, the participants would not be harmed and are likely to have opportunities for } \\
\text { learning. }\end{array}$ \\
\hline 4. Competent & $\begin{array}{l}\text { The focus on context-free rules in the previous stages drains attentional resources and through increasing familiarity with } \\
\text { the teaching process becomes far less necessary. Trainees at this stage move away from a predominant focus on applied } \\
\text { problem solving and the application of learned rules towards an embodied engagement in the moment and a greater } \\
\text { degree of fluid responsiveness. Although there are some inconsistencies and problems, the teaching is at a workable level } \\
\text { of competence and is clearly 'fit for practice'. }\end{array}$ \\
\hline 5. Proficiency & $\begin{array}{l}\text { At this stage, the teacher has an intuitive ability to use learned patterns without decomposing them into component features } \\
\text { and has an increased level of moment-by-moment responsiveness and flexibility. The teacher operates from direct contact } \\
\text { with the arising of experience in self, in individual participants and in the group. }\end{array}$ \\
\hline 6. Advanced & $\begin{array}{l}\text { At this stage, the skill that the teacher has in teaching mindfulness is part of him/her as a person. While teaching, they are } \\
\text { immersed in the process and no longer use rules, guidelines or maxims. She/he has deep tacit understanding of the } \\
\text { teaching and is an original, flexible and fluid teacher. The breadth and depth of knowledge of the teacher at this } \\
\text { developmental stage is an inspiration to others. At this stage, the teacher's skills are consistent even in the face of strong } \\
\text { difficulty such as participant hostility or strong emotion. The process is intuitive, and the teacher is unlikely to be able to } \\
\text { easily articulate how he/she is teaching if asked. }\end{array}$ \\
\hline
\end{tabular}

who has taught a significant number of classes and is now deeply familiar with and at home within the teaching process; and 'advanced' is likely to be a teacher with considerable depth and length of experience and with a maturity to their personal mindfulness and teaching practice. In practice, any one teacher is unlikely to demonstrate consistent levels of competence-some variability between adjacent levels is typical. As we refine our understanding of the processes involved in the development of competencies, we can also more skilfully refine our training processes. For example, is it helpful in the early stages to offer trainees specific guidance on ways of structuring class dialogue or to emphasise the development of process skills such as embodiment and relational presence? It is also unclear whether the different areas of competence develop at different or similar rates and what processes particularly support development in specific areas.

Relating Developmental Stages to Domains of Competence In our development of criteria for assessing mindfulness-based teaching (Crane et al. 2011), we describe six domains within the teaching process (coverage, pacing and organi- sation of session curriculum; relational skills; embodiment of mindfulness; guiding mindfulness practices; conveying course themes through interactive inquiry and didactic teaching; management of group learning environment), and have developed domain-specific descriptors for each level of competence in the adapted framework described in Table 1. Assessment of competence would thus yield a multidimensional profile offering the teacher tailored feedback on teaching strengths and areas for development within the various aspects to the teaching process.

\section{Current Status of Methodologies for Assessing Mindfulness-Based Teaching Competencies}

We now move on to review the range of assessment methodologies currently used in mindfulness-based training and research programmes. Several of the methods described do not directly assess teaching competencies but do assess areas that are thought to be related to their development. No one method holds the distinction of being a complete and 
gold standard, and none is currently empirically supported. It is clear that we have much to learn about the utility of these varying methodologies. In our view, the complexity of mindfulness-based teacher competencies is never going

Table 2 Methodologies for assessing mindfulness-based teaching competence and skills associated with competence

Teaching portfolios

Reflective assignments

Academic assignments

Self-assessment

Peer assessment

Review of teaching by an expert panel via DVD recording or live observation

Use of 'rating scales' to measure adherence and/or competencies
Accumulated experience gathered in the form of a portfolio or dossier which can include detailed narrative of session by session teaching processes including personal reflections on the experience, publicity materials, participant feedback forms and outcomes of evaluation/audit of the class. Teaching portfolios do not provide direct evidence of competencies but in our experience are useful accompaniments to other methodologies.

Given that the teaching process strongly relies on the teacher having a sophisticated capacity to attune to internal experience in the form of sensations, thoughts and emotions, the training programmes represented by the authors all include assessment of the particular style of reflective capacity required for mindfulness-based teaching.

All the postgraduate training programmes represented by the authors require trainees to produce written assignments on the theory and background underpinning MBSR and/or MBCT teaching. Commonly, these require students to synthesise their own experience with the literature on mindfulness-based teaching.

Explicitly inviting trainees to assess their competencies after teaching can be a way of actively engaging them in an exploration of their strengths and areas for development from a different perspective than receiving feedback from others. If trainees develop the capacity to engage in honest reflection of their own teaching, this will serve their ongoing development beyond their engagement in formal training processes.

The usual context for practising skills in the training group is with peer trainees. Peer feedback therefore becomes a key training tool and in itself is a skill which needs training so that the feedback is both honestly and sensitively offered. The process is mutual-in offering feedback, trainees are honing their understanding of the competencies required through a direct experience of what works well and less well.

The Center for Mindfulness in Massachusetts offers 'certification' as an MBSR teacher (Center for Mindfulness in Medicine and Health Care and Society 2011), a process which is open to practitioners who have completed the range of Oasis MBSR training processes and which involves assessment of teaching competence via DVD recording by two experienced MBSR teachers. Although the criteria used for this assessment are not published, it does offer a marker that the teacher has made his/her teaching practice available for external scrutiny and has participated in a robust series of training processes.

All the postgraduate training programmes represented by the authors use review of teaching as an assessment methodology. The 'unit' of assessment is progressively developed during training programmes. In the early stages of training, students are assessed on their skill in teaching elements of the curriculum, whereas at the end, they are required to submit DVDs of an entire 8-week MBCT or MBSR course for review.

Two scales have been developed to measure mindfulness-based teaching adherence and competencies - the MBCT adherence scale (Segal et al. 2002) and the Mindfulness-Based Relapse Prevention Adherence and Competence Scale (MBRP-AC) (Chawla et al. 2010). The MBCT adherence scale (Segal et al. 2002) is a 17-item scale designed to measure the teacher's adherence to the treatment protocol - the scale does not address competence. The assessor carries out the relatively straightforward process of rating the presence/ absence of core features of the approach via DVD observation of the teacher. The MBRP$\mathrm{AC}$ is a measure of treatment integrity for mindfulness-based relapse prevention (MBRP; Chawla et al. 2010). It was developed in the context of a randomized controlled trial and consists of two sections: adherence (adherence to individual components of MBRP and discussion of key concepts) and competence (ratings of therapist style/approach and performance). Assessments are made via audio recordings.

Whilst both these scales have clear utility in the contexts for which they were developed, they have a number of flaws in the context of assessing competence in training programmes. They are both model specific, whereas the training programmes we represent include a diversity of students implementing mindfulness-based approaches in a diversity of contexts; assessments in the context of training programmes need to account for both adherence to programme and teaching competence; the MBRP-AC assesses via audio recordings which does not enable assessment of the 'visible' aspects of the teaching process (nonverbal communication, posture and behaviour); both scales employ assessors who are not themselves teachers - in our view, assessing teaching competence requires proficiency in mindfulness-based teaching so that there is attunement to the subtleties of the process. 
to be encapsulated by any one method of assessment, and it seems important to approach the endeavour from a range of angles (Table 2).

Our explorations and reflections on assessing teaching competencies led us to conclude that our existing assessment processes did not fully meet the needs of the situation. Although we are committed to using a range of assessment methodologies, we are also clear that assessment of teaching is incomplete if it does not include direct observation of the teaching. It is our belief that training programmes which offer trainees credentials for teaching MBSR or MBCT have an ethical duty to include assessment of competencies, so that graduation through the program holds some tangible meaning for the trainee and the general public they will be serving. As a UK forum, we had a surprising degree of consistency of judgement within core teams when making assessments of teaching competence. However, there were a number of challenges. First, the criteria on which assessments were made were not clearly stated, leading to a lack of transparency to trainees and making the basis on which final grades were decided difficult to defend. Second, on a national level, there were no agreed benchmarks for mindfulness-based teaching competencies so trainees, their employers and the general public had nothing against which to relate the awards. Third, in research settings, there is no agreed standard for acceptable levels of MBSR/MBCT teaching with which to judge the adequacy of the mindfulness-based intervention. Therefore, ways of assessing teaching competencies which are valid and reliable need to be developed and researched.

Over the last 3 years, Bangor, Oxford and Exeter Universities, which all conduct mindfulness research, offer postgraduate training in MBSR and/or MBCT, and which all include assessment of competencies in their training programmes, have worked together to develop a system for assessing mindfulness-based teaching competencies: the Bangor, Exeter and Oxford Mindfulness-Based Interventions: Teaching Assessment Criteria (Crane et al. 2011). Our intention has been to pool the expertise of our centres in developing the criteria; to move us towards nationally agreed standards and benchmarks for readiness to teach; and to develop methodologies for assessing mindfulnessbased teaching competencies which in themselves are open to scrutiny and which enable the field to make the transition from the preparation of future teachers being carried out primarily by first- and second-generation teachers.

\section{Conclusions}

We are at a critical juncture in the development of mindfulness-based teaching in secular contexts. People across the world are increasingly finding mindfulness teachings relevant in their lives, and there are numerous ways in which the application of mindfulness could expand in health, education and social care settings. Yet, there are only a limited number of mindfulness teachers who have received substantial and in-depth training from first- and second-generation mindfulness teachers. For those people who are able to offer training, there are imperatives and responsibilities to rise to the challenge so that there are greater numbers of available teachers who can offer MBSR and MBCT with integrity.

It is clearly in the interests of the development of mindfulness-based teaching if coherency about competence, standards and training develops from those teachers with extensive experience of teaching mindfulness themselves and training other mindfulness teachers. Communication of issues related to quality and integrity needs to be compatible with the culture of the mainstream settings in which MBSR and MBCT are offered whilst also honouring of the integrity of the work. There is a risk that a polarity develops between those who are immersed in the teaching of mindfulness and those whose interest in mindfulness is more pragmatic. The work of articulating integrity and quality of teaching clearly bridges these dimensions because it has to be both deeply rooted in the pedagogy and also relevant to and workable within the mainstream contexts within which MBCT and MBSR classes are offered.

Acknowledgements The authors would like to thank the teachers from the Center for Mindfulness, Massachusett for their visionparticularly Ferris Urbanowski, Pam Erdmann, Melissa Blacker, Jon Kabat-Zinn and Saki Santorelli. They have been at the heart of the UK mindfulness developments through their support, friendship, encouragement, teaching and supervision. They have conveyed to us both the spirit and rigour of what must be asked of ourselves and our trainees, and also a clear sense that this vision can become real on the ground in mainstream settings in the UK context. We would also like to thank the teaching staff within our own centres who have contributed to the ideas expressed in this paper over many years - in particular Judith Soulsby, Sarah Silverton, Michael Chaskalson, David Elias, Trish Bartley, Jody Mardula, Annee Griffiths, Taravajra, Karunavira, Vanessa Hope, Eluned Gold, Claire Brejcha, Alison Evans, Christina Feldman, Jenny Wilks, Christina Surawy; and Catrin Eames who brings her research expertise to the question of mindfulness-based teaching competence.

\section{References}

Barber, J. P., Sharpless, B. A., Klostermann, S., \& McCarthy, K. S. (2007). Assessing intervention competence and its relation to therapy outcome: a selected review derived from the outcome literature. Professional Psychology: Research and Practice, 38 (5), 493-500.

Center for Mindfulness in Medicine, Health Care and Society. (2011). Teacher certification review. http://www.umassmed.edu/ $\mathrm{cfm} /$ certification/index.aspx?linkidentifier=id\&itemid $=41322$. Accessed 14 Jun 2011 
Chartered Institute of Personnel and Development. (2011). Competence and competency frameworks. http://www.cipd.co.uk/hrresources/factsheets/competence-competency-frameworks. aspx\#link_4. Accessed 14 Jun 2011

Chawla, N., Collinsa, S., Bowena, S., Hsua, S., Growa, J., Douglass, A., et al. (2010). The mindfulness-based relapse prevention adherence and competence scale: development, interrater reliability, and validity. Psychotherapy Research, 2011(4), 388-397.

Crane, R., Kuyken, W., Hastings, R., Rothwell, N., \& Williams, J. M. G. (2010). Training teachers to deliver mindfulness-based interventions: learning from the UK experience. Mindfulness, 1, 74-86.

Crane, R. S., Soulsby, J. G., Kuyken, W., Williams, J. M. G., \& Eames, C. (2011). The Bangor, Exeter \&Oxford mindfulness-based interventions: teaching assessment criteria. Unpublished manuscript.

Dreyfus, H. L., \& Dreyfus, S. E. (1986). Mind over machine: the power of human intuition and experience in the age of computers. New York: Free Press.

Epstein, R. M., \& Hundert, E. M. (2002). Defining and assessing professional competence. Journal of the American Medical Association, 287, 226-235.

Grossman, P. (2010). Mindfulness for psychologists: paying kind attention to the perceptible. Mindfulness, 1, 87-89.

IAPT. (2011). Improving access to psychological therapies. http:// www.iapt.nhs.uk/. Accessed 8 Jul 2011

Kabat-Zinn, J. (2011). Some reflections on the origins of MBSR, skillful means, and the trouble with maps. Contemporary Buddhism, 11(1).

Kabat-Zinn, J., Santorelli, S., Blacker, M., Brantley, J., Meleo-Meyer, F., Grossman, P., et al. (2011). Training teachers to deliver mindfulness-based stress reduction principles and standards. http://www.umassmed.edu/cfm/trainingteachers/index.aspx. Accessed 18 Mar 2011

Kaslow, N. J. (2004). Competencies in professional psychology. American Psychologist, 59, 774-781. doi:10.1037/0003-066X.59.8.774.

McCown, D., Reibel, D., \& Micozzi, M. S. (2010). Teaching mindfulness: a practical guide for clinicians and educators. New York: Springer.

National Institute for Clinical Excellence (2009). Depression: the treatment and management of depression in adults (update). Clinical Guideline 90. [http://guidance.nice.org.uk/CG90].

National Institute for Clinical Excellence (2004). Depression: management of depression in primary and secondary care (Clinical Guideline No. 23). www.nice.org.uk/CG023NICE guideline.

Owen, S. (1996). An anthology of Chinese literature. New York: WW Norton.
Rodolfa, E., Bent, R., Eisman, E., Nelson, P., Rehm, L., \& Ritchie, P. (2005). A cube model for competency development: implications for psychology educators and regulators. Professional Psychology: Research and Practice, 36(4), 347-354. doi:10.1037/07357028.36.4.347.

Santorelli, S. (1999). Heal thy self. New York: Bell Tower.

Santorelli, S., Goddard, T., Kabat-Zinn, J., Kesper-Grossman, U., \& Reibel, D. (2011). Standards for the formation of MBSR teacher trainers: Experience, qualifications, competency and ongoing development, . Investigating and Integrating Mindfulness in Medicine, Health Care, and Society 9th Annual International Scientific Conference for Clinicians, Researchers and Educators, Boston, USA.

Segal, Z. V., Williams, J. M. G., \& Teasdale, J. D. (2002). Mindfulness-based cognitive therapy for depression: a new approach to preventing relapse. New York: Guilford Press.

Segal, Z. V., Teasdale, J. D., Williams, J. M., \& Gemar, M. C. (2002). The mindfulness-based cognitive therapy adherence scale: interrater reliability, adherence to protocol and treatment distinctiveness. Clinical Psychology \& Psychotherapy, 9(2), 131-138. doi:10.1002/cpp.320.

Sharpless, B. A., \& Barber, J. P. (2009). A conceptual and empirical review of the meaning, measurement, development, and teaching of intervention competence in clinical psychology. Clinical Psychology Review, 29, 47-56.

Skills for Health. (2011). Competences/National occupational standards. http://www.skillsforhealth.org.uk/about-us/competences $\%$ 10national-occupational-standards/. Accessed 15 Jun 2011

Teasdale, J. D., \& Chaskalson, M. (2011). How does mindfulness transform suffering? I: the nature and origins of dukkha. Contemporary Buddhism, 12(01), 89-102. doi:10.1080/ 14639947.2011.564824.

Teasdale, J. D., Segal, Z. V., \& Williams, J. M. G. (2003). Mindfulness training and problem formulation. Clinical Psychology: Science and Practice, 10(2), 157-160.

UK Network of Mindfulness-Based Teacher Trainers. (2010). Good practice guidance for teaching mindfulness-based courses. http://www.bangor.ac.uk/mindfulness/documents/ MBA\%20teacherGPG-Feb\%2010.pdf. Accessed 8 Jun 2011

Weck, F., Bohn, C., Ginzburg, D. M., \& Stangier, U. (2011). Treatment integrity: implementation, assessment, evaluation, and correlations with outcome. Verhaltenstherapie, 21(107), 99. doi: $10.1159 / 000328840$.

Williams, J. M. G. (2008). Mindfulness, depression and modes of mind. Cognitive Therapy and Research, 32, 721-733. doi:10.1007/s10608-008-9204-z. 